\title{
Gait Kinematics in Spastic Hemiplegic Patients - A Descriptive Study
}

\author{
Vipin Kumar Karunakaran ${ }^{1}$, Sreejith Kalathummarathu², Muralidharan P.C. ${ }^{3}$ \\ ${ }^{1}$ Government Medical College, Kottayam, Kerala, India. ${ }^{2}$ Department of Physical Medicine and Rehabilitation, \\ Government Medical College, Kottayam, India. ${ }^{3}$ Department of Physical Medicine and Rehabilitation, \\ Government Medical College, Kozhikode, Kerala, India.
}

\section{ABSTRACT}

\section{BACKGROUND}

Gait impairment in stroke has a significant impact on the quality of life of stroke survivors. Instrumental gait analysis helps to identify gait impairments and determine appropriate treatment strategies and monitor the effectiveness of therapeutic interventions. The objective of the study was to determine the gait kinematics in spastic hemiplegic patients using the Instrumental Gait Analysis (IGA) system.

\section{METHODS}

The present descriptive study was done in the Gait Laboratory of the Department of Physical Medicine and Rehabilitation, Medical College Kottayam. A sample size of 100 subjects was obtained fulfilling the inclusion criteria. Gait analysis was carried out in the gait laboratory using the iSen3.08 system and STT-IWS sensors and kinematic data collected. Descriptive statistical analysis was performed to find out the mean and standard deviation for the quantitative data and qualitative data was expressed as frequency and percentage.

\section{RESULTS}

Reduction in peak hip extension in the late stance phase and peak hip flexion in the swing phase was observed. There was reduced knee flexion during the loading response phase and knee hyperextension at midstance. Knee flexion was reduced during the late stance phase and swing phase. In the majority of subjects, the ankle remained in plantar flexion at initial contact and there was reduced ankle plantar flexion during terminal stance and reduced ankle dorsiflexion in the swing phase.

\section{CONCLUSIONS}

Analysis of hemiplegic gait helps to plan efficient treatment strategies and provides appropriate interventions. The analysis of gait provides insight into the need for various interventional strategies like therapeutic exercises, medical management, neurolysis, orthotic assistances and surgical interventions. This enhances ambulation potential and provides better quality of life.
Corresponding Author: Dr. Muralidharan P.C., Associate Professor, Physical Medicine \& Rehabilitation, Government Medical College, Kozhikode, Kerala, India.

E-mail: muralipc@yahoo.co.in pcmurali35@gmail.com

DOI: $10.14260 / \mathrm{jemds} / 2022 / 68$

How to Cite This Article: Karunakaran VK, Kalathummarathu $S$, Muralidharan PC. Gait kinematics in spastic hemiplegic patients - a descriptive study. I Evolution Med Dent Sci 2022;11(02):352-357, DOI: 10.14260/jemds/2022/68

Submission 20-01-2022,

Peer Review 25-01-2022,

Acceptance 03-02-2022,

Published 05-02-2022.

Copyright (C) 2022 Vipin Kumar Karunakaran et al. This is an open access article distributed under Creative Commons Attribution License [Attribution 4.0 International (CC BY 4.0)]

\section{KEY WORDS}

Stroke, Hemiplegia, Gait, Kinematics. 


\section{BACKGROUND}

Cerebrovascular accident or stroke is a global health problem and is the third leading cause of disability. ${ }^{1}$ Stroke is a nontraumatic brain injury - ischemic (85\%) or hemorrhagic (15 $\%)$ - causing neurological deficits which alter the functional status of the individual. The stroke survivor demonstrates different grades of spasticity, muscle weakness and gait impairment ${ }^{2}$ which significantly alter functional independence ${ }^{3}$ and reduce the quality of life. ${ }^{4}$ About 52 to 85 $\%$ of hemiplegic patients regain their walking ability, but their gait differs from that of normal healthy subjects. The pattern of gait in such a person depends on the severity and extent of involvement, response to the treatment, appropriate rehabilitation interventions, comorbidities and psycho-social factors. Hemiplegic gait is characterized by reduced stride length, gait velocity, cadence and increased energy consumption when compared to normal subjects. ${ }^{5}$ Abnormal patterns in spastic hemiplegia include reduced hip flexion, reduced knee flexion, knee hyperextension during stance and excessive ankle plantar flexion (equinus) during both the swing and stance phase of the gait. 6 The ankle equinus predispose to dynamic knee recurvatum. ${ }^{7}$ This can lead to compensatory manoeuvres like hip circumduction, hip hiking and contralateral vaulting. 8

The term 'gait analysis' refers to the scientific investigation of human locomotion evaluating body movements, mechanics and muscular activity. ${ }^{9}$ Gait analysis is done in hemiplegic patients to identify gait impairments. It is carried out in two ways- observational and instrumental gait analysis. Observational gait analysis is the commonly used method to evaluate gait abnormalities in clinical practice. It is inexpensive but the results are qualitative and observer-dependent. Instrumental gait analysis (IGA) provides three-dimensional information of human walking and is the gold standard for gait evaluation in patients with gait abnormalities. Modern gait analysis systems also quantify spatiotemporal, kinetic, kinematic and electromyographic (EMG) parameters of gait. ${ }^{10}$ Kinetics is the study of internal and external forces acting on the body that causes the motions during gait. Kinematics is the study of the motions of the joints or body segments independent of the internal and external forces that cause them.

The rehabilitation interventions after hemiplegia enable the patients to regain their motor functions and become functionally independent. Interventions to improve gait is an essential component of stroke rehabilitation. This involves measures to prevent or reduce contractures, reduce spasticity, improve power, coordination and balance and strategies to improve propulsion. Conventional physiotherapy measures, robotic management, interventions using botulinum toxins or phenol, orthotic management and surgical measures constitute the various modalities employed for the rehabilitation of a stroke patient. Instrumental gait analysis can become an effective tool in planning and monitoring the effects of various interventions. The need for expensive equipment, time, and availability of qualified personnel for data interpretation limits its use.

In a normal human gait, different segments of the body move with a coordinated rotatory motion.
The temporospatial parameters indicate cadence, walking velocity and stride length. ${ }^{11}$ Kinematics analyses the position and orientation of the body segments and the angular excursions of the joints without considering the forces causing them ${ }^{12}$ during various phases of gait. In the sagittal plane, it measures the angle between hip, knee and ankles. ${ }^{13}$ The gait kinematics in hemiplegic patients differs from normal individuals in both stance and swing phases of gait.

During the stance phase of the gait, post-stroke hemiplegic patients may exhibit a range of motion of the hip that may not be significantly different from normal.14 There may be reduced hip flexion at initial contact or increased hip flexion. ${ }^{15,16}$ There may be increased knee flexion,14,17,18 initially or reduced knee flexion during the early part of stance phase, followed by a knee hyperextension in late stance and delayed movement into knee flexion in preparation for swing. ${ }^{14,16}$ During the stance phase of the gait the ankle joint also exhibits significant variation in the kinematics. On the affected side, the initial contact is typically made with the foot flat or ankle in a plantar-flexed position which results in the toe as the initial contact instead of a heel strike. ${ }^{16}$ During the stance phase after initial contact the ankle has been found to exhibit irregular movements into dorsiflexion, $^{14}$ reduced dorsiflexions in midstance ${ }^{15}$ or increased plantar flexion in the stance phase of the gait. 15

The swing phase patterns of the hip, knee, and ankle motions on the paralyzed side in hemiplegia have been characterized by reduced flexion at the hip ${ }^{15}$ and hiking of the hip, absent or reduced knee flexion ${ }^{15}$ and reduced dorsiflexion or continuous plantar flexion at the ankle. ${ }^{14,15}$

As a result of these variations, the hemiplegic gait has been characterized by a stiff knee during the swing phase. It has been observed that there is a relative increase in the limb length produced by the limited hip and knee flexion and reduced ankle dorsiflexion which results in reduced floor clearance by the affected side during the swing phase. As a result, the patient adopts compensatory patterns in the hemiplegic gait such as dragging of the toes or circumduction of the leg14,15,17 or upward tilt of the hip called hip hiking. ${ }^{19}$

The prevalence of stroke varied between 84-264/100000 in rural areas and 334-424/100000 in urban areas. ${ }^{20}$ With advancements in acute care and early rehabilitation there has been a significant increase in the potential for ambulation and the level of independence of the stroke survivor.

Gait analysis helps the clinicians to identify gait deficits and the compensatory strategies adopted by the hemiplegic patients, which when combined with a neurologic assessment help to identify the causes of deviations and help them to design the best gait training program suitable for their patients. This also provides for various treatment options to improve the gait parameters. The response of a patient to a chosen intervention can be assessed objectively by a laboratory gait assessment. Though analysis of gait is an essential rehabilitation component and research is conducted, there is a paucity of literature regarding kinematics and kinetics in our state. The present study attempted to provide an early understanding of the angular excursions of the hip, knee and ankle in post hemiplegic gait which enables in designing rehabilitation interventions. 


\section{METHODS}

This was a descriptive study carried out in the Gait Laboratory, Department of Physical Medicine and Rehabilitation, Government Medical College, Kottayam from March 2019 to March 2020. The patients who reported to the outpatient clinic were recruited for the study as per the inclusion criteria. A sample size of 100 was obtained based on the observations of Bourdarham et ${ }^{2} .^{21}$ in the kinematic parameters of gait in hemiplegic patients. The standard deviation of stride length was taken from the study for sample size calculation.

$\mathrm{n}=\frac{4 \times S D^{2}}{d^{2}}$

$\mathrm{n}=$ sample size, $\mathrm{SD}=$ standard deviation,

$\mathrm{d}=$ precision $=20 \% \times \mathrm{SD}$

$\mathrm{SD}=0.20$

$\mathrm{SD}^{2}=0.20 \times 0.20=0.04$

$4 \mathrm{SD}^{2}=4 \times 0.04=0.16$

$\mathrm{d}=20 \% \times \mathrm{SD}=20 \% \times 0.2=0.04$

$\mathrm{d}^{2}=0.0016$

$\mathrm{n}=4 \mathrm{SD}^{2} / \mathrm{d}^{2}=0.16 \div 0.0016=100$

We included patients with a 'first stroke' with unilateral hemiplegia, unilateral hemispheric lesions confirmed by computed tomography (CT) or magnetic resonance imaging (MRI), aged 45-65 years with duration of hemiplegia between 1-12 months after the stroke, ability to walk at least 10 meters without any help or assistive devices, ability to understand verbal commands and to cooperate with the experimental procedures and no other diagnosed diseases known to affect walking performances.

We excluded subjects with a previous history of severe diseases of heart, lung, liver, kidney etc., patients with concomitant neurological diseases, stroke due to traumatic brain injury and tumour, patients with impaired comprehension, aphasia and cognitive disorders and noncooperative patient/ caregiver.

The study was conducted after obtaining ethical clearance from the Institutional Review Board. Informed consent was obtained in the prescribed format from patients. Confidentiality and anonymity of the patients' information were maintained during and after the study.

Data was collected from each patient and recorded in a pre-prepared proforma.

The kinematic data of hemiplegic patients were recorded by a motion capture system and multiple sensors. STT-IWS inertial sensors were used in an iSen system to track the movement of individual body segments and transfer realtime data to the software.

The sensors were placed on thighs, legs and feet and one at the mid pelvis. The subjects were required to walk barefoot at their natural velocity. The distance covered in one direction was 5 meters. The joint movements were captured by the sensors and the virtual image of joint motion was recorded in the computer using the iSen3.08 application.
One gait cycle was taken for assessment. That is from the initial contact of the diseased limb to the next initial contact of the same limb.

The sagittal plane joint excursions (hip flexion and extension, knee flexion and extension and ankle dorsiflexion and plantar flexion) were analysed during each phase of the gait cycle.

\section{Statistical Analysis}

Data were summarized in an excel sheet. Descriptive statistics were performed to find out the mean and standard deviation for demographic and outcome variables. Quantitative data were expressed in mean and standard deviation and qualitative data in frequency and percentage.

\section{RESULTS}

At initial contact, the mean hip angle was $+11.24^{\circ}$ with a standard deviation (SD) of 7.11 and the mean knee angle was $+2.5^{\circ}$ with a standard deviation of 4.53 and the mean ROM at the ankle was $-4.30^{\circ}$ with a standard deviation of 5.44 .

During the Loading Response phase, the mean hip angle was $+4.78^{\circ}(\mathrm{SD}=5.35)$, the mean knee angle was $+1.93^{\circ}(\mathrm{SD}=$ 6.31 ) and the mean angle at the ankle was $-4.79^{\circ}(\mathrm{SD}=4.28)$.

At the midstance phase, the mean hip ROM was $-0.34^{\circ}$ $(\mathrm{SD}=4.63)$, the mean knee angle was $-1.13^{\circ}(\mathrm{SD}=4.53)$ and the mean angle at the ankle was $+0.25^{\circ}(\mathrm{SD}=3.51)$.

At the terminal stance, the mean hip angle was $-11.90^{\circ}$ $(\mathrm{SD}=6.62)$. The mean knee angle was $+10.23^{\circ}(\mathrm{SD}=6.99)$ and the mean angle at the ankle joint was $-5.71^{\circ}(\mathrm{SD}=4.24)$.

At the pre-swing phase, the mean hip angle was $-5.37^{\circ}$ $(\mathrm{SD}=7.99)$, the mean angle at the knee joint was $+21.30^{\circ}(\mathrm{SD}=$ $7.43)$ and the mean angle at the ankle was $-14.30^{\circ}(\mathrm{SD}=7.56)$.

At the mid-swing phase, the mean angle at the hip joint was $+10.30^{\circ}(\mathrm{SD}=8.66)$, the mean angle at the knee joint was $+20.47^{\circ}(\mathrm{SD}=13.85)$ and the mean angle at the ankle joint was $-5.06^{\circ}(\mathrm{SD}=6.60)$.

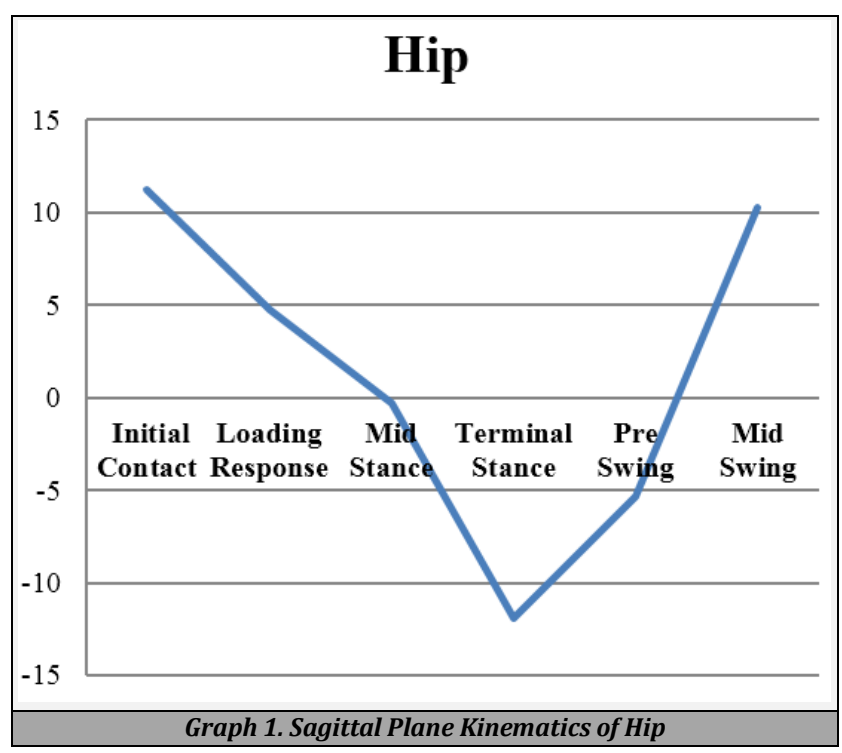



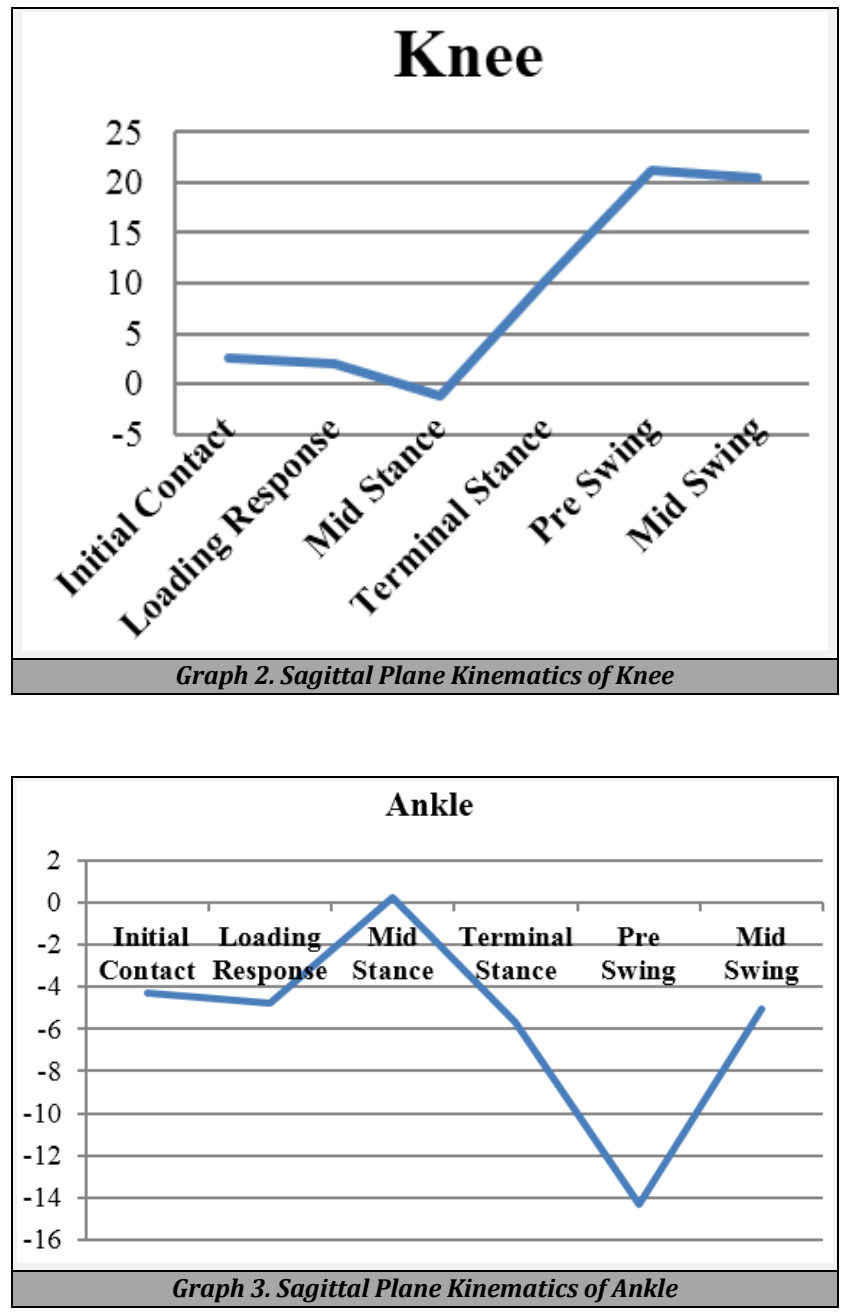

\begin{tabular}{|c|c|c|c|c|}
\hline \multirow{3}{*}{$\begin{array}{l}\text { Phases } \\
\text { Initial } \\
\text { contact }\end{array}$} & \multirow{3}{*}{$\begin{array}{c}\text { Location } \\
\text { Ankle }\end{array}$} & \multicolumn{2}{|c|}{ Deviation } & \multirow{2}{*}{$\begin{array}{c}\text { Frequency } \\
74\end{array}$} \\
\hline & & Toe contact & Abnormal & \\
\hline & & Heel contact & Normal & 26 \\
\hline \multirow{2}{*}{ Mid stance } & \multirow{2}{*}{ Knee } & Hyperextension & Abnormal & 60 \\
\hline & & Flexion & Normal & 40 \\
\hline \multirow{2}{*}{ Mid swing } & \multirow{2}{*}{ Ankle } & Plantar flexion & Abnormal & 73 \\
\hline & & Dorsiflexion & Normal & 27 \\
\hline
\end{tabular}

$74 \%$ of the subjects had ankle plantar flexion at initial contact. In $60 \%$ of the subjects, there was knee hyperextension at mid-stance and $73 \%$ of the subject's ankles were plantar flexed at mid-swing.

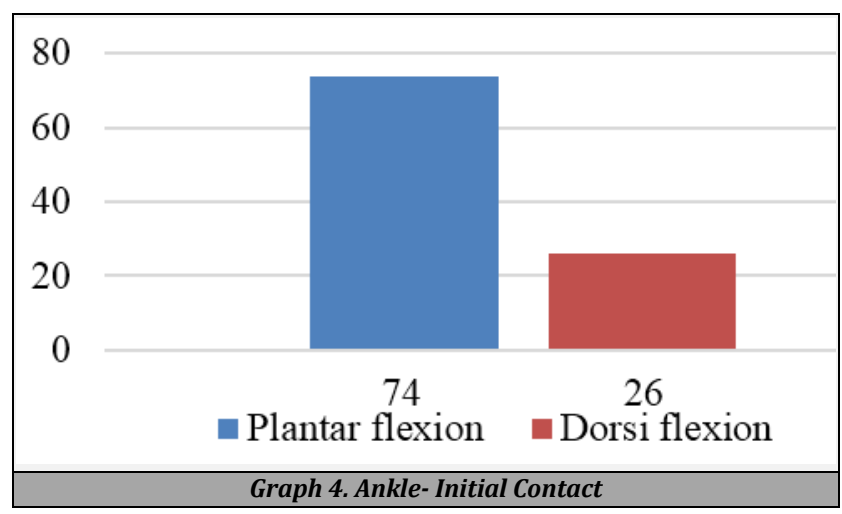

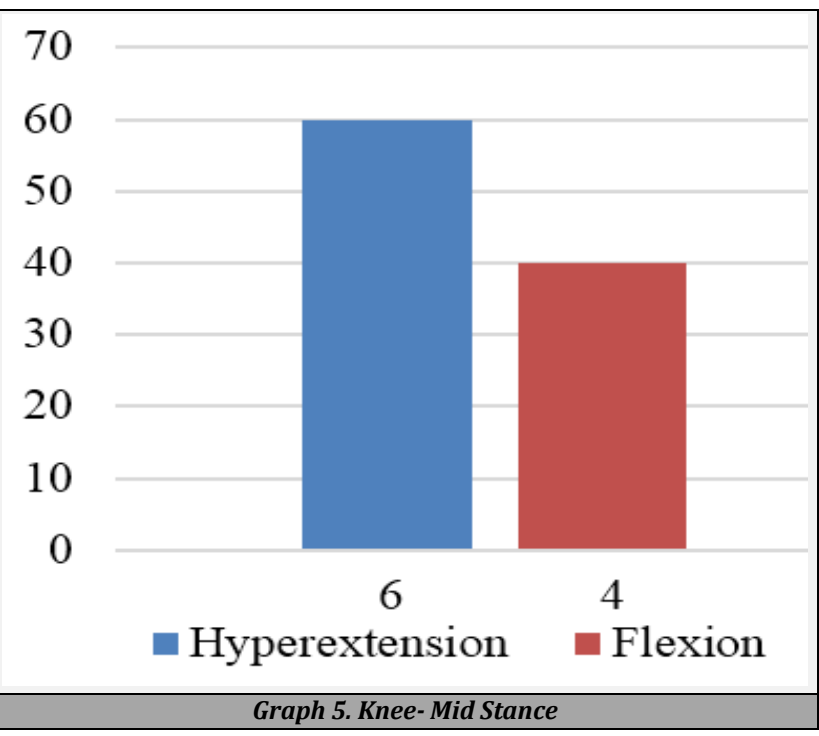

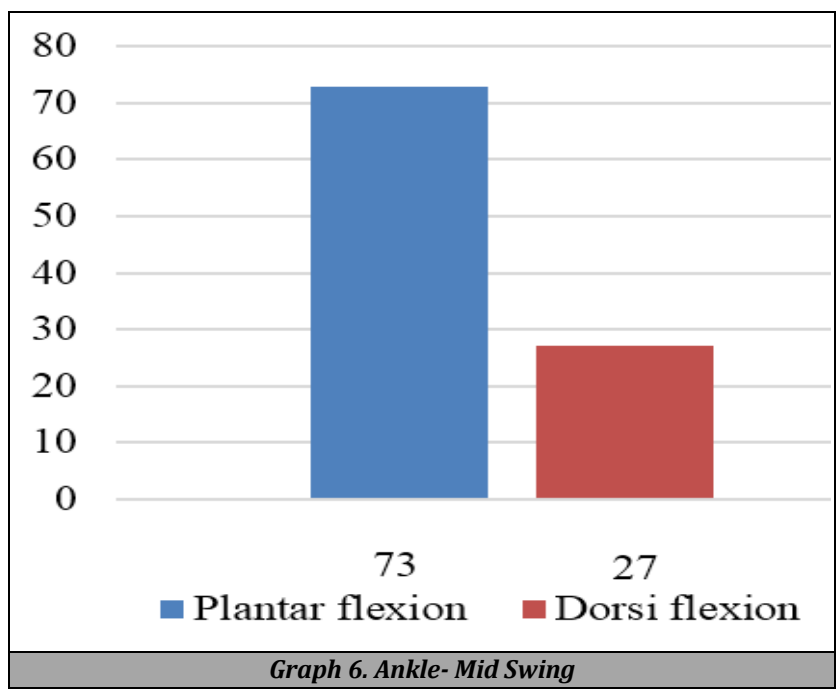

\section{DISCUSSION}

In the present study, the mean hip angle of the affected side at initial contact was $+11.24^{\circ}$. The maximum hip flexion at initial contact was $+32.05^{\circ}$. In $68 \%$ of the subjects, the hip flexion at initial contact was $<15^{\circ}$. In $6 \%$ of the subjects, the hip remained in extension at initial contact. The mean hip extension at terminal stance was $-11.90^{\circ}$ and at mid-swing, the mean hip ROM was $10.30^{\circ}$.

Kramers et al. $^{6}$ in their study reported reduced hip extension in the stance phase and delay in the initiation of hip flexion during the pre-swing phase. The hip joint ROM in the affected side showed a significant reduction in the sagittal plane when compared to the unaffected side. The decreased hip extension has been a commonly reported kinematic deviation following hemiplegic stroke, 22 this may be due to reduced power of the hip extensor muscles, hip flexor contracture or spasticity of the hip flexors. Ankle plantar flexor spasticity in the stance phase also contributes to a reduced hip extension during the late stance phase. ${ }^{22}$ The reduced hip flexion during mid-swing may be attributed to the reduced power of the rectus femoris and iliopsoas muscle which tends to accelerate the limb in the swing phase.23 During the stance phase, the sagittal plane hip ROM exhibited 
a greater variety of atypical joint motions in hemiplegic patients. Reduced or increased hip flexion has been reported at initial contact. The magnitude of peak hip extension during the stance phase, especially at the late stance and push-off, is reduced relative to normal subjects and the hip may sometimes be flexed at toe-off. ${ }^{24}$ The observations from our study correlate well with these observations.

Straudi S et al. ${ }^{25}$ in their low functioning walking cluster group found that hip motion reflected a slight flexion reduction instance $\left(20.5^{\circ}\right)$ and a lack of extension in the late stance phase $\left(-2.4^{\circ}\right)$. In the present study, mean hip extension at the terminal stance phase was $-11.90^{\circ}$. The difference may be due to the larger sample size in the present study. Our study did not stratify subjects as low, medium and high functioning walking groups.

In the present study, the mean knee ROM at initial contact was found to be $2.51^{\circ}$. One-fourth of the subjects had knee hyperextension at the initial contact. The mean knee ROM at loading response was $1.93^{\circ}$. There was a reduced flexion during the loading response phase and in $42 \%$ of the subjects, there was knee hyperextension. At mid-stance, the mean knee ROM was $-1.13^{\circ}$ and $60 \%$ of the patient's knees went into hyperextension. At the late stance, the knee went into flexion but the mean ROM was reduced $\left(10.23^{\circ}\right.$ and $21.30^{\circ}$ at the terminal stance and pre-swing respectively). The mean knee flexion at the swing phase was $20.47^{\circ}$. The hyperextension of the knee in the early part of the stance phase was due to the weakness of the quadriceps or spasticity of ankle plantar flexors. ${ }^{22}$ The reduced knee flexion during the swing phase may be attributed to the reduced power of the hamstrings, spasticity of the quadriceps and reduced hip flexion and ankle dorsiflexion ${ }^{23}$ due to which the patient may adopt some compensatory strategies like circumduction, hip hiking or contralateral vaulting. ${ }^{26}$

Jiang $\mathrm{X}$ et al. ${ }^{27}$ in their quantitative analysis of knee abnormalities in hemiplegic subjects observed that the ROM at initial contact on the affected side was $4.49^{\circ}$ and maximum extension in the stance phase on the affected side was $-0.89^{\circ}$. These results are comparable with the present study.

Straudi S, in the low functioning walking group, identified a stiff-knee pattern with reduced flexion both in early stance $\left(1.0^{\circ}\right)$ and swing phases $\left(13.7^{\circ}\right)$. In the intermediatefunctioning walkers, the knee joint had a normal flexion during loading response $\left(10.4^{\circ}\right)$ with a slight hyperextension in the stance phase $\left(-5.2^{\circ}\right)$ and in the swing phase, the knee flexion was reduced $\left(24.5^{\circ}\right)$. In the high functioning walkers, the knee flexion during loading response was normal $\left(11.2^{\circ}\right)$ and hyperextension in midstance $\left(-11.0^{\circ}\right)$. Knee flexion during the swing phase was also reduced $\left(39.0^{\circ}\right)$. Our study also demonstrates hyperextension in the stance phase and reduction in the knee flexion during the swing phase. Since our study does not divide the study subjects into different functional walking groups, a comprehensive comparison is not possible. Kramers et al. and Carmo et al. also observed reduced knee flexion during the swing phase.

In the present study, the mean ankle ROM at initial contact was $-4.30^{\circ}$, at loading response $-4.79^{\circ}$, mid-stance $+0.25^{\circ}$, terminal stance $-5.71^{\circ}$, pre-swing $-14.30^{\circ}$ and at midswing $-5.06^{\circ}$. In $76 \%$ of the subjects, the ankle remained in plantar flexion at initial contact. A plantar flexed ankle will result in a forefoot contact or toe strike instead of a heel strike. This may be due to weakness of the tibialis anterior and other dorsiflexors or due to gastrosoleus spasticity or tendon Achilles contracture.22,26 The relative ankle dorsiflexion at midstance was decreased because of the reduced knee flexion at loading response $\left(1.93^{\circ}\right)$ which rotated the tibia forward over the fixed foot. At pre-swing, the ankle ROM was $-14.30^{\circ}$, negative indicating plantar flexion, which was found to be slightly reduced. This may be due to the inability to produce sufficient active tension with the ankle plantar flexor muscles in a late stance. ${ }^{26} \mathrm{At}$ mid-swing also the mean ankle ROM was $-5.06^{\circ}$. This may be due to excessive ankle plantar flexor spasticity or ankle dorsiflexor weakness. A plantar flexed ankle will hinder the toe clearance which will lead the patient to adopt the compensatory strategies.

In a study conducted by Kesikburun $\mathrm{S}$ et $\mathrm{al}^{28}$ in hemiplegic patients the mean ankle ROM at initial contact was $-6.80^{\circ}$ and at mid-swing was $-5.69^{\circ}$, comparable with our study. Kramer in his study also observed reduced ankle dorsiflexion during the swing phase. Straudi S in their study reported reduced dorsiflexion $\left(5.0^{\circ}\right)$ in the mid-stance phase. In the study by Pongpipatpaiboon $\mathrm{K}$ et al. $^{29}$ in hemiplegic patients, the mean ankle ROM from mid-stance to mid-swing was $-10.4^{\circ}$. These results correlate with our observations. In their study, Pongpipatpaiboon $\mathrm{K}$ et al. observed that the use of AFO reduced the mean ankle ROM to $-6.1^{\circ}$ which was statistically significant, and augmented the toe clearance and reduced compensatory movements during the swing phase of the gait.

\section{CONCLUSIONS}

It was found that the hip moved in a narrow range during the different phases of gait. There was reduced knee flexion at the loading response phase and knee hyperextension at midstance. During the late stance phase and swing phase, the knee flexion was found to be reduced. The ankle remained in plantar flexion at initial contact, in the majority of cases leading to a forefoot strike. The ankle plantar flexion was reduced during the terminal stance. The ankle dorsiflexion was found to be reduced during the swing phase. Hence it can be concluded that in hemiplegic patients there would be a reduction in the mean range of motion of hip, knee and ankle in the sagittal plane in both stance and swing phase of the gait. Various rehabilitation interventions are undertaken based on the clinical and functional status of the patient as evidenced by gait analysis. This signifies the role of instrumental gait analysis in planning and monitoring treatment strategies in post-stroke hemiplegic patients.

\section{Limitations}

In the present study, there was no control group. We did not compare the hemiplegic side with the unaffected side. Kinetic analysis and spatiotemporal parameters were not considered. There were no follow-up studies.

\section{Further Scope}

Further analysis can be undertaken to assess the efficacy of treatment strategies. 


\section{REFERENCES}

[1] Fergin VL, Norrving B, Mensa GA. Global burden of stroke. Circ Res 2017;120(3):439-48.

[2] Ribeiro T, Britto H, Oliveira D, et al. Effects of treadmill training with partial body weight support and the proprioceptive neuromuscular facilitation method on hemiparetic gait: a randomized controlled study. Eur J Phys Rehabil Med 2013;49(4):451-61.

[3] Cui C, Bian GB, Hou ZG, et al. simultaneous recognition and assessment of post-stroke hemiparetic gait by fusing kinematic, kinetic and electrophysiological data. IEEE Trans Neural Syst Rehabil Eng 2018;26(4):856-64.

[4] King RB. Quality of life after stroke. Stroke 1996;27(9):1467-72.

[5] Chen G, Patten C, Kothari D, et al. Gait differences between individuals with post-stroke hemiparesis and non-disabled controls at matched speeds. Gait Posture 2005;22(1):51-6.

[6] De Quervain IA, Simon SR, Leurgans S, et al. Gait pattern in the early recovery period after stroke. JBJS 1996;78(10):1506-14.

[7] Rose J, Gamble JG. Human walking. $3^{\text {rd }}$ edn. Philadelphia: Lippincott Williams \& Wilkins 2006: p. 234.

[8] Kerrigan DC, Deming LC, Holden MK. Knee recurvatum in gait: a study of associated knee biomechanics. Arch Phys Med Rehabil 1996;77(7):645-50.

[9] Wernick J, Volpe R. Lower extremity function and normal mechanics. Chap- 1. In: Valmassy $\mathrm{R}$, ed. Clinical biomechanics of the lower extremities. St Louis: Mosby 1996.

[10] Fang J, Mu Z, Xu Z, et al. Models for temporal-spatial parameters in walking with cadence ratio as the independent variable. Med Biol Eng Comput 2019;57(4):877-86.

[11] Mikos V, Yen SC, Tay A, et al. Regression analysis of gait parameters and mobility measures in a healthy cohort for subject-specific normative values. PLoS One 2018;13(6):e0199215.

[12] Soutas-Little RW. Motion analysis and biomechanics. Chap- 2. De Lisa JA, ed. Gait analysis in the science of rehabilitation. Scientific and Technical Publication 1998.

[13] Esquenazi A, Talaty M. Gait analysis, technology and clinical applications. Phys Med Rehabil 2011:99-116.

[14] Richards C, Knutsson E. Evaluation of abnormal gait patterns by intermittent-light photography and electromyography. Scand J Rehabil Med 1974;3:61-8.
[15] Lehmann JF, Condon SM, Price R, et al. Gait abnormalities in hemiplegia: their correction by ankle-foot orthoses. Arch Phys Med Rehabil 1987;68(11):763-71.

[16] Pinzur MS, Sherman R, DiMonte-Levine P, et al. Gait changes in adult onset hemiplegia. Am J Phys Med 1987;66(5):228-37.

[17] Olney SJ, Colborne GR, Martin CS. Joint angle feedback and biomechanical gait analysis in stroke patients: a case report. Phys Ther 1989;69(10):863-70.

[18] Trueblood PR, Walker JM, Perry J, et al. Pelvic exercise and gait in hemiplegia. Phys Ther 1989;69(1):18-26.

[19] Takebe K, Basmajian JV. Gait analysis in stroke patients to assess treatments of foot-drop. Arch Phys Med Rehabil 1976;57(1):305-10.

[20] Pandian JD, Sudhan P. Stroke epidemiology and stroke care services in India. J Stroke 2013;15(3):128-34.

[21] Boudarham J, Roche N, Pradon D, et al. Variations in kinematics during clinical gait analysis in stroke patients. PLoS One 2013;8(6):e66421.

[22] Moseley A, Wales A, Herbert R, et al. Observation and analysis of hemiplegic gait: stance phase. Aust J Physiother 1993;39(4):259-67.

[23] Moore S, Schurr K, Wales A, et al. Observation and analysis of hemiplegic gait: swing phase. Aust J Physiother 1993;39(4):271-8.

[24] Richards C, Knutsson E. Evaluation of abnormal gait patterns by intermittent-light photography and electromyography. Scand J Rehabil Med Suppl 1974;3:61-8.

[25] Straudi S, Manca M, Aiello E, et al. Sagittal plane kinematic analysis of the six-minute walk test: A classification of hemiplegic gait. Eur J Phys Rehabil Med 2009;45(3):341-7.

[26] Frontera WR, Delisa JA. Human walking. Chap- 5. In: Delisa's physical medicine \& rehabilitation: principles and practice. $5^{\text {th }}$ edn. Lippincott Williams \& Wilkins 2010:121-39.

[27] Jiang X, Hu X. Analysis of the knee angle in hemiplegic gait. Chin J Rehabil Med 2007;22(10):918-20.

[28] Kesikburun S, Yavuz F, Güzelküçük Ü, et al. Effect of ankle foot orthosis on gait parameters and functional ambulation in patients with stroke. Turk J Phys Med Rehabil. 2017;63(2):143-8.

[29] Pongpipatpaiboon K, Mukaino M, Matsuda F, et al. The impact of ankle-foot orthoses on toe clearance strategy in hemiparetic gait: a cross-sectional study. J NeuroEngineering Rehabil 2018;15(1):41. 